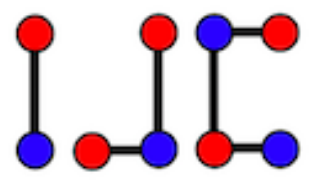

\title{
Decomposition of complete graphs into connected unicyclic graphs with eight edges and pentagon
}

\author{
Dalibor Froncek ${ }^{\mathrm{a}}$, O’Neill Kingston ${ }^{\mathrm{b}}$ \\ ${ }^{a}$ Department of Mathematics and Statistics, University of Minnesota Duluth, Duluth, MN, U.S.A. \\ ${ }^{b}$ Department of Mathematics, Iowa State University, Ames, IA, U.S.A. \\ dalibor@d.umn.edu, oneillk@iastate.edu
}

\begin{abstract}
A $G$-decomposition of the complete graph $K_{n}$ is a family of pairwise edge disjoint subgraphs of $K_{n}$, all isomorphic to $G$, such that every edge of $K_{n}$ belongs to exactly one copy of $G$. Using standard decomposition techniques based on $\rho$-labelings, introduced by Rosa in 1967, and their modifications we show that each of the ten non-isomorphic connected unicyclic graphs with eight edges containing the pentagon decomposes the complete graph $K_{n}$ whenever the necessary conditions are satisfied.
\end{abstract}

Keywords: Graph decomposition, $\rho$-labeling, unicyclic graph, pentagon

Mathematics Subject Classification: 05C51, 05 C78

DOI: $10.19184 /$ ijc.2019.3.1.3

\section{Introduction}

A decomposition of the complete graph $K_{n}$ is a family of pairwise edge disjoint subgraphs $\mathcal{D}=\left\{G_{0}, G_{1}, \ldots, G_{s}\right\}$ such that every edge of $K_{n}$ belongs to exactly one member of $\mathcal{D}$. If each subgraph $G_{r}$ is isomorphic to a given graph $G$ we speak about a $G$-decomposition of $K_{n}$, or a $G$-design. The decomposition is cyclic if there exists an ordering $\left(x_{1}, x_{2}, \ldots, x_{n}\right)$ of the vertices of $K_{n}$ and isomorphisms $\phi_{r}: G_{0} \rightarrow G_{r}, r=0,1,2, \ldots, s$, such that $\phi_{r}\left(x_{i}\right)=x_{i+r}$ for each $i=1,2, \ldots, n$. The subscripts are taken modulo $n$. Similarly, the decomposition is one-rotational if there exists an ordering $\left(x_{1}, x_{2}, \ldots, x_{n}\right)$ of the vertices of $K_{n}$ and isomorphisms $\phi_{r}: G_{0} \rightarrow G_{r}$,

Received: 23 Jan 2019, Revised: 03 Mar 2019, Accepted: 07 Jun 2019. 
$r=0,1,2, \ldots, s$, such that $\phi_{r}\left(x_{i}\right)=x_{i+r}$ for each $i=1,2, \ldots, n-1$ and $\phi_{r}\left(x_{n}\right)=x_{n}$. The subscripts here are taken modulo $n-1$.

A graph $G$ is unicyclic if it contains exactly one cycle. In this paper, we provide the necessary and sufficient conditions for decompositions of complete graphs into each of the ten connected unicyclic graphs with eight edges containing the pentagon and prove that each of them decomposes the complete graph $K_{n}$ whenever the necessary conditions are satisfied.

We will use standard decomposition methods based on $\rho$-labelings, introduced by Rosa [8] as well as some other slightly modified well-known tools.

\section{Related results}

It seems that there has been no attempt to completely classify graphs with 8 vertices and 8 edges decomposing complete graphs. Between the time the results contained in this paper were obtained and prepared for publication, a partial result on four particular connected graphs with pentagon was published in [10]. The methods used in the paper were mostly design theory based and are significantly different from methods that we use in this paper. Therefore, for the sake of completeness, we include our results for those graphs as well.

At the same time, there are several papers on decompositions of $K_{n}$ into graphs with 8 edges and 5,6 or 7 vertices.

This section will summarize what is known about classification of smaller graphs, that is, graphs where $\mid(E(G) \mid \leq 8$ or $\mid(V(G) \mid \leq 8$ that form decompositions of complete graphs. An exhaustive search was conducted, and the following results were found, however, due to the limited availability of some journals there could be other results not summarized here.

Graphs with five vertices and eight edges were examined by Colbourn, Ge, and Ling in 2008, due to their applicability with respect to the problem of grooming traffic in optical networks [3]. There are only two non-isomorphic graphs with five vertices and eight edges, shown in Figure 1.
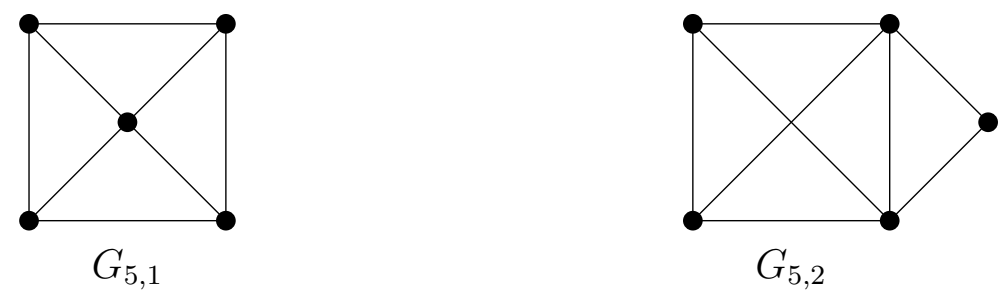

Figure 1: Connected graphs with 8 edges and 5 vertices

Colbourn, Ge and Ling proved the following results for the graphs $G_{5,1}$ and $G_{5,2}$.

Theorem 2.1 (Colbourn, Ge, Ling 2008). There exists a decomposition of $K_{n}$ into $G_{5,1}$ if and only if $n \equiv 0(\bmod 16)$ except possibly when $n=32$ or $n=48$.

Theorem 2.2 (Colbourn, Ge, Ling 2008). There exists a isomorphic decomposition of $K_{n}$ for $G_{5,2}$ if and only if $n \equiv 0,1(\bmod 16)$ except when $n=16$ and possibly when $n=48$. 
Kang, Yuan, and Liu researched graphs with six vertices and eight edges in 2005 [7]. There are 22 non-isomorphic graphs of this type, and they proved the following theorem with respect to decompositions of complete graphs.

Theorem 2.3 (Kang, Yuan, Liu 2005). Let $G$ be a connected graph with six vertices and eight edges. Then $G$ forms a decomposition of $K_{n}$ if and only if $n \equiv 0,1(\bmod 16)$ and $n \geq 16$ with two possible exceptions for $n=32$.

We were unable to find any results on graphs with 8 edges and 7 vertices.

For graphs with 8 edges and 8 vertices, Kang and Zhang determined the spectrum completely for the four graphs shown in Figure 2.
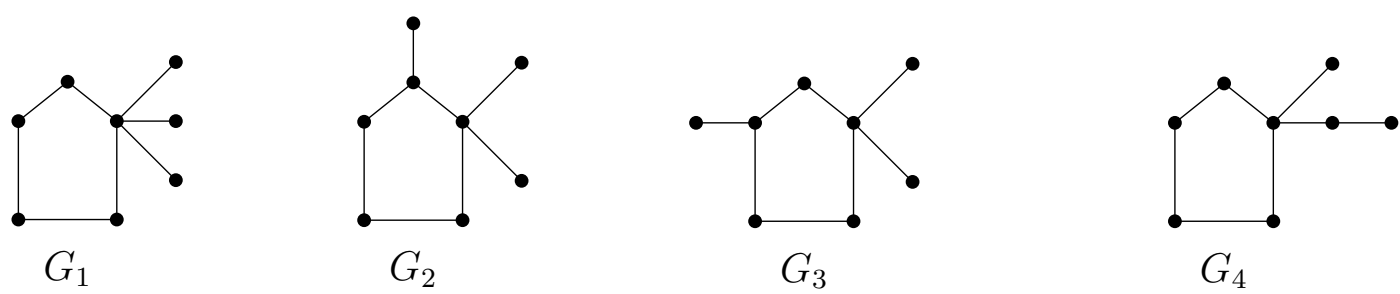

Figure 2: Graphs $G_{1}, G_{2}, G_{3}, G_{4}$ by Kang and Zhang

Theorem 2.4 (Kang, Zhang 2015). Let $G_{i}$ be a connected graph with eight vertices and eight edges shown in Figure 2. Then $G_{i}$ forms a decomposition of $K_{n}$ if and only if $n \equiv 0,1(\bmod 16)$ and $n \geq 16$.

Bipartite connected unicyclic graphs with 8 edges and 8 vertices other than $C_{8}$ were studied by Fahnenstiel and Froncek in [5]; $C_{8}$ decompositions exist if and only if $n \equiv 1(\bmod 16)$ as proved by Rosa [8].

Theorem 2.5 (Fahnenstiel, Froncek 2017). Let $G$ be a connected bipartite unicyclic graph with eight vertices and eight edges other than $C_{8}$. Then $G$ forms a decomposition of $K_{n}$ if and only if $n \equiv 0,1(\bmod 16)$ and $n \geq 16$.

\section{Tools and methods}

The major tool we use is the $\rho$-labeling, first defined by Rosa [8], although he originally used the term valuation.

Definition 3.1. Let $G$ be a graph with $n$ edges. A $\rho$-labeling of $G$ is an injection $f: V(G) \rightarrow$ $\{0,1, \ldots, 2 n\}$ inducing the length function $\ell: E(G) \rightarrow\{1,2, \ldots, n\}$ defined as

$$
\ell(u v)=\min \{|f(u)-f(v)|, 2 n+1-|f(u)-f(v)|\}
$$

with the property that

$$
\{\ell(u v): u v \in E(G)\}=\{1,2, \ldots, n\}
$$


A more restrictive version is the $\sigma$-labeling, also introduced by Rosa in [8].

Definition 3.2. Let $G$ be a graph. A $\sigma$-labeling of $G$ is a $\rho$-labeling such that $\ell(u v)=|f(u)-f(v)|$.

Rosa [8] proved that if a graph $G$ with $n$ edges has one of the above labelings, then a decomposition of a complete graph $K_{2 n+1}$ exists.

Theorem 3.1 (Rosa 1967). A cyclic decomposition of the complete graph $K_{2 n+1}$ into subgraphs isomorphic to a given graph $G$ with $n$ edges exists if and only if there exists a $\rho$-labeling of the graph $G$.

The above labelings enable isomorphic decompositions of complete graphs of odd order, but similar methods exist for complete graphs of even order under certain circumstances. It is well known that certain $\rho$-labeled graphs can form isomorphic decompositions of $K_{2 n k}$ (see, e.g., [4]). Although the proof of the theorem is well-known, we repeat it here for the reader's convenience.

Theorem 3.2. Let $G$ be a graph with $n$ edges and let $v$ be a vertex of degree 1 in $G$. If $G-v$ has a $\rho$-labeling, then $G$ forms a one-rotational isomorphic decomposition of $K_{2 n}$.

Proof. Let $u v$ be the only edge incident with vertex $v$ in $G$. We denote the vertices of $K_{2 n}$ as $x_{1}, x_{2}, \ldots, x_{2 n}$.

Because $G-v$ allows a $\rho$-labeling, by Theorem 3.2 there exists a cyclic decomposition of $K_{2 n}-x_{2 n}$ into $2 n-1$ copies of the graph $G-v$. Call them $G_{1}^{\prime}, G_{2}^{\prime}, \ldots, G_{2 n-1}^{\prime}$. We can assume without loss of generality that in the $i$-th copy of $G-v, G_{i}^{\prime}$, the vertex $u$ is mapped onto $x_{i}$, and identify $v$ with $x_{2 n}$. Then for each $i=1,2, \ldots, 2 n-1$, the edge $x_{i} x_{2 n}$ of $K_{2 n}$ appears exactly in one copy of $G-v$, namely in $G_{i}^{\prime}$. Obviously, adding the edge $x_{i} x_{2 n}=u v$ to the appropriate $G_{i}^{\prime}$, we obtain a $G$-decomposition of $K_{2 n}$. The length of $u v$ will be denoted by $\infty$.

For bipartite graphs, Rosa also found another labeling (called $\alpha$-labeling) that allows decompositions of larger complete graphs, namely $K_{2 n k+1}$ for any positive integer $k$. This labeling cannot be used for graphs with odd cycles. However, one can observe that all unicyclic graphs are almost bipartite, that is, we can obtain a bipartite graph by removing a single edge. Blinco, El-Zanati, and Vanden Eynden [1] provide a similar tool, $\gamma$-labeling, that allows decomposition of $K_{2 n k+1}$ into almost bipartite graphs. Even more powerful labeling for tripartite graphs, called tripartite $\rho$-labeling, was later found by Bunge, Chantasartrassmee, El-Zanati, and Vanden Eynden [2].

It would be probably possible to find a $\gamma$ - or tripartite $\rho$-labeling for most or all of the graphs we are focusing on, but even then we would have to use another method for decompositions of $K_{2 n k}$. Therefore, we decided to use a different approach that will cover decompositions of both $K_{2 n k+1}$ and $K_{2 n k}$ concurrently.

We will find a $\sigma$-labeling for a graph $H$ satisfying assumptions of Theorem 3.2 that can be decomposed into $k$ copies of $G$, where $G$ is a connected unicyclic graph with 8 edges containing a pentagon. Because we use a $\sigma$-labeling rather than $\rho$-labeling, the lengths of edges are always computed as $\ell(u w)=|f(u)-f(w)|$ regardless of whether we are using the lengths with respect to $K_{2 n+1}$ or $K_{2 n-1}$ and we can replace the longest edge $u v$ by the edge of length $\infty$.

We formalize the method in the following observation.

Proposition 3.3. Let $H$ be a graph decomposable into $k$ copies of a graph $G$ and there exists an $H$-decomposition of $K_{m}$. Then there also exists a $G$-decomposition of $K_{m}$. 


\section{Graphs with pentagon}

The necessary condition for $K_{n}$ to be decomposable into $k$ copies of a graph with 8 edges is that $8 k$ divides $n(n-1) / 2$, the number of edges in $K_{n}$. This is only true when $n \equiv 0,1(\bmod 16)$.

First we provide a catalog of all connected unicyclic graphs on 8 edges with a pentagon. By $G\left(b_{1}, \ldots, b_{5} ; t\right)$ we denote such graph with trees on $b_{i}$ edges attached to some of the five vertices of the pentagon; the parameter $t$ just distinguishes between non-isomorphic graphs with the same five-tuple $b_{1}, \ldots, b_{5}$.
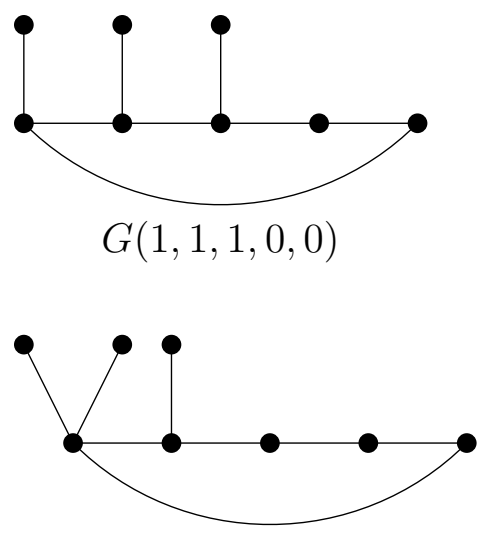

$G(2,1,0,0,0 ; s)$

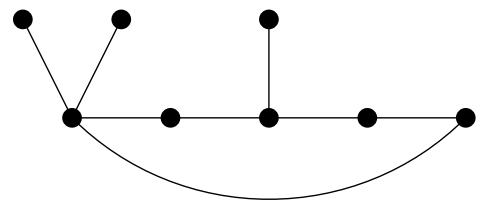

$G(2,0,1,0,0 ; s)$
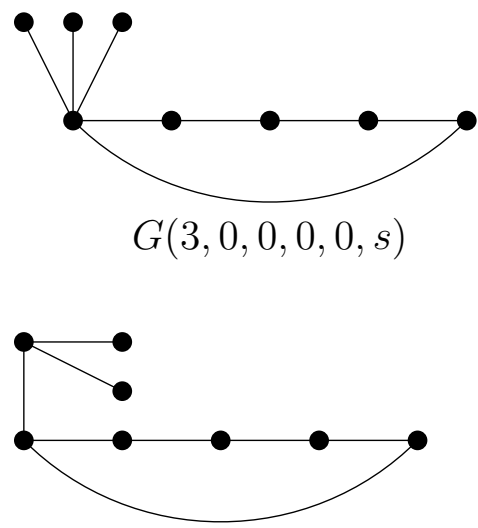

$G(3,0,0,0,0, f)$
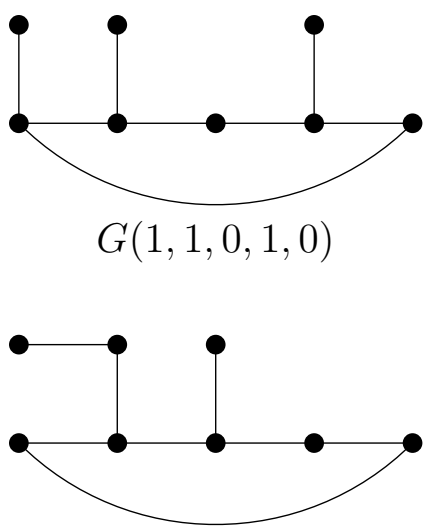

$G(2,1,0,0,0 ; p)$

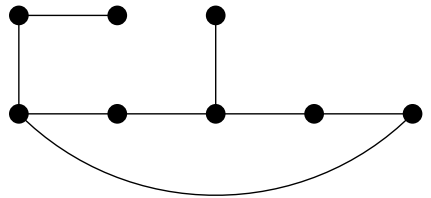

$G(2,0,1,0,0 ; p)$

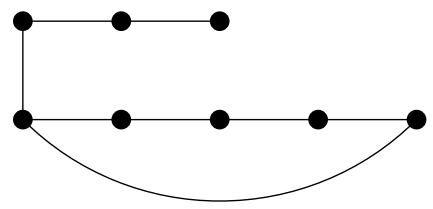

$G(3,0,0,0,0, p)$

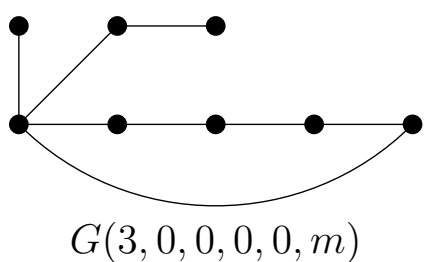

Figure 3: Connected unicyclic graphs with 8 edges and pentagon 
Now for every $k \geq 1$ and each $G\left(b_{1}, b_{2}, \ldots, b_{5} ; t\right)$ we need to find a graph $H_{k}\left(b_{1}, \ldots, b_{5} ; t\right)$ with $8 k$ edges such that

(i) $H_{k}\left(b_{1}, b_{2}, \ldots, b_{5} ; t\right)$ is decomposable into $k$ edge-disjoint copies of $G\left(b_{1}, b_{2}, \ldots, b_{5} ; t\right)$,

(ii) $H_{k}\left(b_{1}, b_{2}, \ldots, b_{5} ; t\right)$ allows a $\sigma$-labeling,

(iii) the longest edge of length $8 k$ has an end-vertex of degree one, and

(iv) the largest label we use is $16 k-2$.

While for $n=16 k+1$ we could use even labels $16 k-1$ and $16 k$ (which is the largest label in $K_{16 k+1}$ ), for $n=16 k$ the vertex $x_{16 k}$ is labeled $\infty$ and for $x_{1}, x_{2}, \ldots, x_{16 k-1}$ we use labels $0,1, \ldots, 16 k-2$.

Construction 4.1 $(k \geq 2)$. We first label the pentagons with one attached edge as shown in Figure 4. Vertex labels are in blue, edge lengths in red. Notice that in the $k$-th copy $G_{k}$ the largest label is not exceeding $16 k-2$, which is the maximum allowed in $K_{16 k}$.

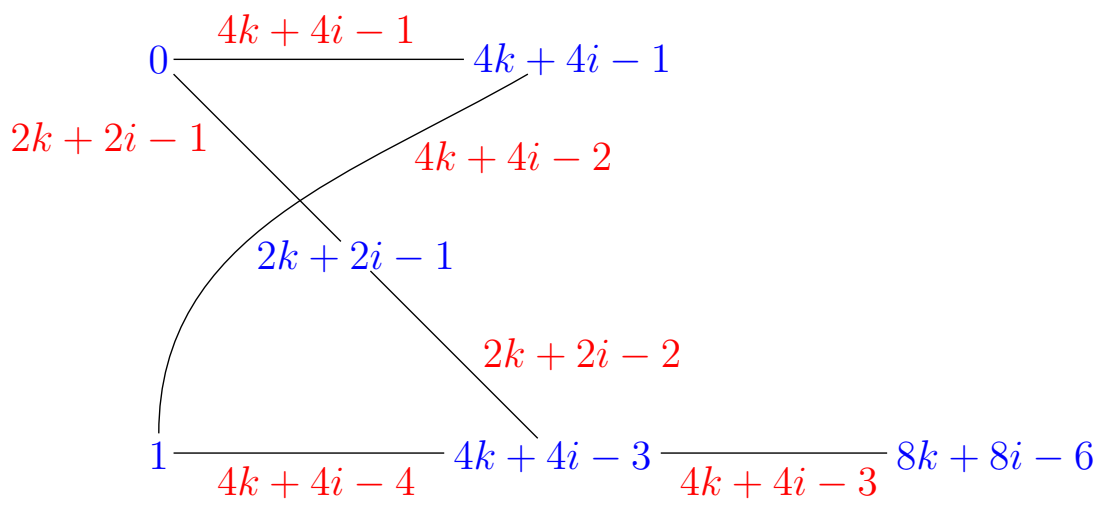

Figure 4: Pentagon with pendant edge in $k$-th copy

The range of edge lengths used in these subgraphs is from $2 k$ to $8 k-1$. The remaining lengths $1,2, \ldots, 2 k-1$ and $8 k$ will be used for the additional edges.

Now we list the remaining edges for the particular graphs. Notice that while the first $k-1$ graphs $G_{1}, G_{2}, \ldots, G_{k-1}$ always follow the same pattern, the last copy $G_{k}$ is different, since we need the longest edge to have one end-vertex of degree one. When $k=1$, we just disregard the first two rows and read only the rows for $G_{k}$.

The asterisk denotes that the vertex label or edge length needs to be replaced by $\infty$ for decompositions of $K_{16}$. 
Graph $G(1,1,1,0,0)$

\begin{tabular}{|c|c|c|c|}
\hline$G(1,1,1,0,0)$ & vertex label & vertex label & edge length \\
\hline$G_{i}, i \leq k-1$ & $2 k+2 i-1$ & $2 k+3 i$ & $i+1$ \\
\hline$G_{i}, i \leq k-1$ & 0 & $k+i$ & $k+i$ \\
\hline$G_{k}$ & $4 k-1$ & $4 k$ & 1 \\
\hline$G_{k}$ & 0 & $8 k^{*}$ & $8 k^{*}$ \\
\hline
\end{tabular}

Graph $G(1,1,0,1,0)$

\begin{tabular}{|c|c|c|c|}
\hline$G(1,1,1,0,0)$ & vertex label & vertex label & edge length \\
\hline$G_{i}, i \leq k-1$ & $4 k+4 i-1$ & $4 k+5 i$ & $i+1$ \\
\hline$G_{i}, i \leq k-1$ & 0 & $k+i$ & $k+i$ \\
\hline$G_{k}$ & 1 & 2 & 1 \\
\hline$G_{k}$ & 0 & $8 k^{*}$ & $8 k^{*}$ \\
\hline
\end{tabular}

Graphs $G(2,1,0,0,0)$

\begin{tabular}{|c|c|c|c|}
\hline$G(2,1,0,0,0 ; s)$ & vertex label & vertex label & edge length \\
\hline$G_{i}, i \leq k-1$ & $2 k+2 i-1$ & $2 k+3 i$ & $i+1$ \\
\hline$G_{i}, i \leq k-1$ & $4 k+4 i-3$ & $5 k+5 i-3$ & $k+i$ \\
\hline$G_{k}$ & $4 k-1$ & $4 k$ & 1 \\
\hline$G_{k}$ & $8 k-3$ & $16 k-3^{*}$ & $8 k^{*}$ \\
\hline
\end{tabular}

\begin{tabular}{|c|c|c|c|}
\hline$G(2,1,0,0,0 ; p)$ & vertex label & vertex label & edge length \\
\hline$G_{i}, i \leq k-1$ & $2 k+2 i-1$ & $2 k+3 i$ & $i+1$ \\
\hline$G_{i}, i \leq k-1$ & $7 k+6 i-5$ & $8 k+7 i-5$ & $k+i$ \\
\hline$G_{k}$ & $16 k-6$ & $16 k-7$ & 1 \\
\hline$G_{k}$ & $4 k-1$ & $12 k-1^{*}$ & $8 k^{*}$ \\
\hline
\end{tabular}

Graphs $G(2,0,1,0,0)$

\begin{tabular}{|c|c|c|c|}
\hline$G(2,0,1,0,0 ; s)$ & vertex label & vertex label & edge length \\
\hline$G_{i}, i \leq k-1$ & 0 & $i+1$ & $i+1$ \\
\hline$G_{i}, i \leq k-1$ & $4 k+4 i-3$ & $5 k+5 i-3$ & $k+i$ \\
\hline$G_{k}$ & $8 k-1$ & $8 k$ & 1 \\
\hline$G_{k}$ & $8 k-3$ & $16 k-3^{*}$ & $8 k^{*}$ \\
\hline
\end{tabular}

\begin{tabular}{|c|c|c|c|}
\hline$G(2,0,1,0,0 ; p)$ & vertex label & vertex label & edge length \\
\hline$G_{i}, i \leq k-1$ & $2 k+2 i-1$ & $2 k+4 i-1$ & $i+1$ \\
\hline$G_{i}, i \leq k-1$ & $7 k+6 i-5$ & $8 k+8 i-6$ & $k+i$ \\
\hline$G_{k}$ & $16 k-6$ & $16 k-7$ & 1 \\
\hline$G_{k}$ & $4 k-1$ & $12 k-1^{*}$ & $8 k^{*}$ \\
\hline
\end{tabular}


Graphs $G(3,0,0,0,0)$

\begin{tabular}{|c|c|c|c|}
\hline$G(3,0,0,0,0 ; s)$ & vertex label & vertex label & edge length \\
\hline$G_{i}, i \leq k-1$ & $4 k+4 i-3$ & $4 k+3 i-4$ & $i+1$ \\
\hline$G_{i}, i \leq k-1$ & $4 k+4 i-3$ & $3 k+3 i-3$ & $k+i$ \\
\hline$G_{k}$ & $8 k-3$ & $8 k-4$ & 1 \\
\hline$G_{k}$ & $8 k-3$ & $16 k-3^{*}$ & $8 k^{*}$ \\
\hline
\end{tabular}

\begin{tabular}{|c|c|c|c|}
\hline$G(3,0,0,0,0 ; f)$ & vertex label & vertex label & edge length \\
\hline$G_{i}, i \leq k-1$ & $8 k+8 i-6$ & $8 k+7 i-7$ & $i+1$ \\
\hline$G_{i}, i \leq k-1$ & $8 k+8 i-6$ & $7 k+7 i-6$ & $k+i$ \\
\hline$G_{k}$ & $16 k-6$ & $16 k-7$ & 1 \\
\hline$G_{k}$ & $16 k-6$ & $8 k-6^{*}$ & $8 k^{*}$ \\
\hline
\end{tabular}

\begin{tabular}{|c|c|c|c|}
\hline$G(3,0,0,0,0 ; m)$ & vertex label & vertex label & edge length \\
\hline$G_{i}, i \leq k-1$ & $4 k+4 i-3$ & $4 k+3 i-4$ & $i+1$ \\
\hline$G_{i}, i \leq k-1$ & $8 k+8 i-6$ & $7 k+7 i-6$ & $k+i$ \\
\hline$G_{k}$ & $8 k-3$ & $8 k-4$ & 1 \\
\hline$G_{k}$ & $16 k-6$ & $8 k-6^{*}$ & $8 k^{*}$ \\
\hline
\end{tabular}

For the last graph $G(3,0,0,0,0 ; p)$ where the path is attached to the pentagon at its end-vertex, the labeling shown in Figure 4 needs to be changed slightly. Namely, the vertex labeled $2 k+2 i-1=3$ needs to be labeled $2 k+2 i-2=2$ instead. The edges incident with that vertex just swap their lengths, as we now have edges 02 of length 2 and 25 of length 3 rather than 03 of length 3 and 35 of length 2 .

\begin{tabular}{|c|c|c|c|}
\hline$G(3,0,0,0,0 ; p)$ & vertex label & vertex label & edge length \\
\hline$G_{i}, i \leq k-1$ & $8 k+8 i-6$ & $7 k+7 i-6$ & $k+i$ \\
\hline$G_{i}, i \leq k-1$ & $7 k+7 i-6$ & $7 k+8 i-5$ & $i+1$ \\
\hline$G_{k}$ & $16 k-6$ & $16 k-5$ & 1 \\
\hline$G_{k}$ & $16 k-5$ & $8 k-5^{*}$ & $8 k^{*}$ \\
\hline
\end{tabular}

Thus we have shown that for every connected unicyclic graph $G\left(b_{1}, b_{2}, \ldots, b_{5} ; t\right)$ on eight edges with a pentagon and every $k \geq 1$ there exists a corresponding graph $H\left(b_{1}, b_{2}, \ldots, b_{5} ; t\right)$ decomposable into $k$ copies of $G\left(b_{1}, b_{2}, \ldots, b_{5} ; t\right)$ with a $\sigma$-labeling and longest edge having an end-vertex of degree one.

Our main result now follows easily.

Theorem 4.1. Let $G$ be a connected unicyclic graph with eight vertices and eight edges where the unique cycle is a pentagon. Then $G$ forms a decomposition of $K_{n}$ if and only if $n \equiv 0,1(\bmod 16)$ and $n \geq 16$. 
Proof. By Construction 4.1, for every connected unicyclic graph on eight edges with a pentagon $G\left(b_{1}, b_{2}, \ldots, b_{5} ; t\right)$ and every $k \geq 1$ there exists a corresponding graph $H\left(b_{1}, b_{2}, \ldots, b_{5} ; t\right)$ decomposable into $k$ copies of the graph $G\left(b_{1}, b_{2}, \ldots, b_{5} ; t\right)$. Moreover, each such $H\left(b_{1}, b_{2}, \ldots, b_{5} ; t\right)$ has a $\sigma$-labeling and the longest edge has an end-vertex of degree one.

Thus, by Theorems 3.1 and 3.2, each $H\left(b_{1}, b_{2}, \ldots, b_{5} ; t\right)$ decomposes $K_{n}$ for every $n \equiv 0,1$ $(\bmod 16)$ and $n \geq 16$. Because each $H\left(b_{1}, b_{2}, \ldots, b_{5} ; t\right)$ is decomposable into $k$ copies of the corresponding graph $G\left(b_{1}, b_{2}, \ldots, b_{5} ; t\right)$, the result now follows by Proposition 3.3.

\section{Conclusion}

In this paper, we have taken further steps to the complete characterization of graphs with eight edges decomposing complete graphs. We have completely solved the problem of decomposition of the complete graph $K_{n}$ into connected unicyclic graphs with eight edges and the unique cycle having length five.

In an earlier paper, Fahnenstiel and Froncek [5] solved analogical problem for connected bipartite unicyclic graphs with eight edges. The remaining cases of unicyclic graphs with eight edges, both connected and disconnected, have been recently solved by Freyberg and Froncek [6]. Thus, the class of unicyclic graphs with eight edges has been completely classified.

\section{References}

[1] A. Blinco, S. El-Zanati, C. Vanden Eynden, On the cyclic decomposition of complete graphs into almost-bipartite graphs, Discrete Math. 284(1-3) (2004), 71-81.

[2] R. Bunge, A. Chantasartrassmee, S. El-Zanati, C. Vanden Eynden, On cyclic decompositions of complete graphs into tripartite graphs, J. Graph Theory 72 (2013), 90-111.

[3] C. Colbourn, G. Ge, A. Ling, Graph designs for the eight-edge five-vertex graphs, Discrete Math. 309 (2009), 6440-6445.

[4] S. El-Zanati, C. Vanden Eynden, On Rosa-type labelings and cyclic graph decompositions, Math. Slovaca 59(1) (2009), 1-18.

[5] J. Fahnenstiel, D. Froncek, Decomposition of complete graphs into connected bipartite unicyclic graphs with eight edges, to appear in Electron. J. Graph Theory Appl.

[6] B. Freyberg, D. Froncek, Decomposition of complete graphs into unicyclic graphs with eight edges, submitted.

[7] Q. Kang, L. Yuan, S. Liu, Graph Designs for all Graphs with Six Vertices and Eight Edges, Acta Math. Appl. Sin. (Engl. Ser.), 21(3) (2005), 469-484.

[8] A. Rosa, On certain valuations of the vertices of a graph, Theory of Graphs (Intl. Symp. Rome 1966), Gordon and Breach, Dunod, Paris, 1967, 349-355. 
[9] J. Yin, B. Gong, Existence of $G$-designs with $|V(G)|=6$, In: W.D. Wallis et al (eds). Combinatorial designs and applications, Marcel Dekker, New York, 1990, 201-218.

[10] Y. Zhang, Q. Kang, Decompositions of $K_{v}$ into four kinds of graphs with eight vertices and eight edges, J. Combin. Math. Combin. Comput. 94 (2015), 157-165. 